Methods: Systematic search strategies were developed for 5 databases (Medline, Embase, Psyclnfo, Scopus, Web of Science) to retrieve studies that investigated the relationship between weather conditions and chronic pain. Original articles describing observational studies that related chronic pain (primary outcome) to weather conditions (exposure), were included. Study characteristics, methodology (e.g. measurements of pain and weather, aspects of statistical analysis) and data on risk of bias (validation of participants' exposure and diagnosis, coverage of weather variation, correction for confounders) was extracted. Methodological rigour was summarised by mapping the methodological variability among studies and ranking these from least to most rigorous.

Results: The searches returned 16,081 articles. After removing 3090 duplicates and excluding 12726 articles during title screening, 265 abstracts were assessed for eligibility. Of 64 observational studies that met the inclusion criteria, 30 $(47 \%)$ investigated pain associated with musculoskeletal conditions, 24 (38\%) investigated headache and $10(16 \%)$ investigated pain associated with other conditions such as sickle cell disease and dental pain. After full text assessment, 10 of 30 papers on musculoskeletal conditions were excluded because they investigated risk of acute pain episodes rather than chronic pain symptoms $(n=6)$, or investigated multiple conditions $(n=4)$. The 20 included studies investigated rheumatoid arthritis $(6,30 \%)$, osteoarthritis $(6,30 \%)$, fibromyalgia $(6,30 \%)$ and low back pain $(2,10 \%)$. A total of 15 studies (75\%) reported some effect of weather on chronic pain, and this was clinically significant (consistent and sufficient size of effect) in 6. Participant numbers varied from 19 to 2491 . 1 study was cross-sectional and 19 longitudinal with follow ups ranging from two weeks to 3.5 years, with participants scoring their pain once to daily for 12 months. Pain was measured with a Visual Analog Scale or Numeric Rating Scale in 17 (85\%). Weather conditions were retrieved from local weather stations, with most studies assuming that participants stayed in the area/city (65\%) or postcode area $(20 \%)$ where they lived.

In $12(60 \%)$ studies, participants were blinded to the study hypothesis. Methods for correcting for confounders were highly variable, with 7 studies not addressing any, and the remainder addressing one or more of 32 confounding variables.

Conclusions: Methodological variability of studies investigating the relationship between weather and chronic musculoskeletal pain is high. This methodological review will inform best research practice for those investigating the relationship between the weather and chronic pain.

Acknowledgements: Mary Ingram, librarian

Disclosure of Interest: None declared

DOI: 10.1136/annrheumdis-2017-eular.6571

\section{AB1119 A DESCRIPTIVE STUDY OF GOUT PATIENTS IN A MULTI- ETHNIC SOCIETY}

C. Chua ${ }^{1}$, P. Cheung ${ }^{2}$, A. Santosa ${ }^{2}$, A. Lim ${ }^{2}$, G.G. Teng ${ }^{2} .{ }^{1}$ Internal Medicine Residency; ${ }^{2}$ Division of Rheumatology, University Medicine Cluster, National University Health System, Singapore, Singapore

Background: Gout is a common inflammatory arthritis with rising global prevalence and health burden, especially in the Asia-pacific regions. Ethnicity may play a significant role suggesting lifestyle and/or genetic predisposition, but studies in Asia are limited ${ }^{1,2}$.

Objectives: Our study sought to assess the demographic and clinical factors of gout in the multi-ethnic Singapore and describe the burden and treatment pattern in our patients.

Methods: 282 adults with rheumatologist-diagnosed gout were recruited from rheumatology clinics of an academic medical centre in Singapore. Data on demographic and lifestyle features, medical conditions, gout severity and treatment were obtained.

Results: 282 subjects were recruited and $92.6 \%$ were men. There were $77 \%$ Chinese, $18.8 \%$ Malays and $2.5 \%$ Indians, compared to Singapore's population makeup $(74 \%, 13 \%$ and $9 \%$ respectively). Mean age at recruitment was 52.6 years (SD 16.1) while age at gout onset was 42.5 years (SD 16.7). $34.4 \%$ received primary or no education; $67.0 \%$ were employed and $20.7 \%$ retired. $22.7 \%$ were current alcohol drinkers while $50.7 \%$ were teetotalers. $23.4 \%$ were current smokers. Mean body mass index (BMI) was $28.1 \mathrm{~kg} / \mathrm{m}^{2}$ (SD 6.0), $26.6 \%$ had $\mathrm{BMI} \geq 30 \mathrm{~kg} / \mathrm{m}^{2}$ while $69.9 \%$ had BMI $\geq 25 \mathrm{~kg} / \mathrm{m}^{2}$. Prevalence of hypertension was $56.7 \%$, diabetes mellitus $18.8 \%$, dyslipidemia $48.2 \%$ and chronic kidney disease (CKD, defined as glomerular filtration rate $<60 \mathrm{ml} / \mathrm{min} / 1.73 \mathrm{~m}^{2}$ ) $32.4 \%$. Malays had significantly less alcohol intake (1.9\%) but higher proportions of diabetes $(32.1 \%)$, CKD $(50.9 \%)$ and obesity $(54.7 \%)$, compared with Chinese subjects $(26.3 \%, 16.1 \%, 28.6 \%$ and $19.8 \%$ respectively, all Ps $<0.05)$. Gout severity was rated moderate by $30.9 \%$ and severe in $50.4 \% ; 69.3 \%$ suffered $\geq 3$ attacks in 6 months. Mean SU was $477.8 \mu \mathrm{mol} / \mathrm{L}$ (SD 130.8). $80.1 \%$ were on allopurinol. Subjects $(22.3 \%)$ who achieved serum urate (SU) $\leq 360 \mu \mathrm{mol} / \mathrm{L}$, when compared to those with $\mathrm{SU}>360 \mu \mathrm{mol} / \mathrm{L}$, were more likely to be on urate lowering therapy ( $82.5 \%$ vs $60.7 \%, \mathrm{P}=0.001)$, on higher mean allopurinol dose [337 mg/d (SD 166) vs $233 \mathrm{mg} / \mathrm{d}$ (SD 140), $P<0.001]$ and statin ( $54.8 \%$ vs $33.3 \%$, $\mathrm{P}=0.003)$. There were no significant differences amongst ethnicities for SU levels, gout severity and number of attacks.

Conclusions: Gout has substantial health burden in Singapore. Hypertension, dyslipidemia and obesity are more prevalent in our gout subjects compared to our population. Despite notably less alcohol intake compared with other cohorts ${ }^{3-5}$, Singapore Malays seemed to suffer higher prevalence and comorbidities of gout.'
Majority of patients had moderate to severe disease but less than 25\% achieved target SU levels highlighting suboptimal management of gout locally.

\section{References:}

[1] Kuo CF, et al. Global epidemiology of gout: prevalence, incidence and risk factors. Nat Rev Rheumatol 2015;11(11):649-62.

[2] Krishnan E, et al. Gout in ambulatory care settings in the United States. J Rheumatol 2008;35(3):498-501.

[3] Lee $\mathrm{CH}$, et al. Factors associated with gout in South Koreans: analysis using the National Health Insurance Corporation and the National Health Screening Exam databases. Clin Rheumatol 2013;32(6):829-37.

[4] Cea Soriano L, et al. Contemporary epidemiology of gout in the UK general population. Arthritis Res Ther 2011;13(2):R39.

[5] Vázquez-Mellado J, et al. Metabolic Syndrome and Ischemic Heart Disease in Gout. J Clin Rheumatol 2004;10(3):105-09.

Disclosure of Interest: None declared

DOI: 10.1136/annrheumdis-2017-eular.3950

\section{AB1120 COMPARISON OF NON-RADIOGRAPHIC SPONDYLOARTHRITIS VERSUS ANKYLOSING SPONDYLITIS PATIENTS UNDERGOING BIOLOGICAL THERAPY}

C. Cobilinschi, D. Opris-Belinski, R. Ionescu. Sfanta Maria Clinical Hospital, Bucharest, Romania

Background: Axial spondyloarthritis include non-radiographic spondyloarthritis (nr-SpA) and ankylosing spondylitis (AS); they reveal the extent of sacroiliitis assessed by conventional $\mathrm{x}$-ray or MRI (1). The natural history of nr-SpA follows various evolution patterns; smoking, male gender, high levels of inflammatory markers or initial radiographic lesions are among predictor factors of progression to AS (2).

Objectives: The objective of the present study was to compare features related to progression of nr-SpA patients versus AS patients undergoing biological therapy with an anti-TNF agent.

Methods: This was an observational, cross-sectional study including 94 patients with $\mathrm{nr}-\mathrm{SpA}$ and AS under continuous anti-TNF therapy for at least six months. SPSS 20.0 was used to analyze data with a $P$ value of 0.05 .

Results: Out of the selected study group, 69 patients were diagnosed with AS having a mean age of $44.8 \pm 10.8$, while 25 patients had $n r-S p A$. Patients with nr-SpA were aged $32.1 \pm 6.6$ years old and $40 \%$ of them were women, a rate significantly than in the $A S$ group $(P=0.05)$. Mean age at disease onset was 30.7 years for the AS subgroup versus only 23.8 years for patients with $\mathrm{nr}-\mathrm{SpA}(\mathrm{P}<0.001)$. AS patients presented a significantly higher value of the BMI compared to $\mathrm{nr}-\mathrm{SpA}$ (27 versus $24.7 \mathrm{~kg} / \mathrm{m}^{2}, \mathrm{P}<0.001$ ). $91 \%$ of patients had positive HLA B27 and $7.4 \%$ had a positive family history of SpA, with no significant differences between the two subgroups. A higher level of CRP was noticed in AS patients $(P=0.038)$.In the study cohort the time interval from symptom onset to establishing a diagnosis was of $39.9 \pm 55.6$ months, with a mean delay interval of 2.32 years for patients with $\mathrm{nr}-\mathrm{SpA}$ and higher, of 3.5 for patients with AS $(\mathrm{P}=0.01)$.

Conclusions: The nr-SpA group had a considerably higher percentage of females compared to the AS subgroup. AS patients presented higher values of CRP at follow-up visits as opposed to nr-SpA patients. There were no significant differences between nr-SpA and AS patients regarding BASDAI or PtGA scores, smoking status or frequency of uveitis. The presence of HLA B27 did not differ between the two subgroups, thus it might not be a reliable predictive factor of progression.

\section{References:}

[1] Akgul O, Ozgocmen S. Classification criteria for spondyloarthropathies. World J Orthop. 2011;2(12):107-115.

[2] Wendling D, Non-radiographic spondyloarthritis: A theoretical concept or a real entity? Jt Bone Spine 2012;79(6):531-533. doi:10.1016/j.jbspin.2012.06.009. Disclosure of Interest: None declared

DOI: 10.1136/annrheumdis-2017-eular.5344

\section{AB1121 TRABECULAR BONE SCORE COMBINED WITH CLINICAL RISK FACTORS CAN PREDICT INCIDENT FRACTURE IN RHEUMATOID ARTHRITIS PATIENTS}

D. Kim ${ }^{1}$, S.-K. Cho ${ }^{1}$, H.-R. Park ${ }^{1}$, Y.Y. Choi ${ }^{2}$, Y.-K. Sung ${ }^{1} .{ }^{1}$ Rheumatology, Hanyang University Hospital for Rheumatic Diseases; ${ }^{2}$ Nuclear medicine, Hanyang University Hospital, Seoul, Korea, Republic Of

Background: Fracture is one of the most common and important comorbidities in rheumatoid arthritis (RA) patients, especially patients who use glucocorticoids (GC). However, bone mineral density (BMD) by dual-energy $\mathrm{x}$-ray absorptiometry (DXA) which is the gold standard of diagnosing and monitoring osteoporosis is not a useful tool for predicting new fracture in RA patients. Previous studies suggested the possibility of trabecular bone score (TBS) as a useful predictor for incident fracture.

Objectives: We aimed to evaluate the accuracy of TBS combined with clinical risk factors or BMD for prediction of new fracture in patients with RA.

Methods: A total of 100 female RA patients were enrolled with assessment of TBS, BMD, and clinical risk factors for fracture. During follow-up period, we 